\title{
Heavy Mesons and Impact Ionization of Heavy Quarkonia *
}

\author{
David Blaschke ${ }^{1,2}$, Yuri Kalinovsky ${ }^{3}$, and Valery Yudichev ${ }^{2}$ \\ 1 Fachbereich Physik, Universität Rostock, D-18051 Rostock, Germany ${ }^{\dagger}$ \\ 2 Bogoliubov Laboratory for Theoretical Physics, Joint Institute for Nuclear \\ Research, 141980 Dubna, Russia \\ 3 Laboratory of Information Technologies, Joint Institute for Nuclear Research, \\ 141980 Dubna, Russia
}

\begin{abstract}
At the chiral restoration/deconfinement transition, most hadrons undergo a Mott transition from being bound states in the confined phase to resonances in the deconfined phase. We investigate the consequences of this qualitative change in the hadron spectrum on final state interactions of charmonium in hot and dense matter, and show that the Mott effect for D-mesons leads to a critical enhancement of the $\mathrm{J} / \psi$ dissociation rate. Anomalous $\mathrm{J} / \psi$ suppression in the NA50 experiment is discussed as well as the role of the Mott effect for the heavy flavor kinetics in future experiments at the LHC. The status of our calculations of heavy quarkonium dissociation cross sections due to quark and gluon impact is reviewed, and estimates for in-medium effects due to the lowering of the ionisation threshold are given.
\end{abstract}

\section{Introduction}

Heavy quarks as constituents of heavy quarkonia and heavy mesons play a decisive role in the diagnostics of hot and dense matter created in ultrarelativistic heavy-ion collisions. The most promiment example is the celebrated $\mathrm{J} / \psi$ suppression effect as a possible signal of the formation of a which was suggested by Matsui and Satz [1] in 1986 and has triggered experimental programs at CERN, Brookhaven and the future GSI facility as well as a broad spectrum of theoretical work ever since. In the present lecture, we will elucidate some aspects of the physics of heavy quarks in a hot and dense medium which demonstrate why $\mathrm{J} / \psi$ suppression is so difficult to interprete. We suggest that $\Upsilon$ may be a rather clean probe of the plasma state to be produced at CERN-LHC.

The production of heavy quark pairs is a hard process well separated from the soft physics governing the evolution of a quark gluon plasma in heavy-ion collisions. However, the formation of heavy quarkonia and heavy mesons as well as their final state interactions may be well modified by a surrounding hot medium. The basic principle of their use as indicators is similar to the situation in Astrophysics where information about the temperature, density, composition and other properties of stellar atmospheres is obtained from an analysis of the modification of emission and absorption spectra relative to terrestrial conditions [2].

^ Published in: Lect. Notes Phys. 647:366-375,2004

† Presently at: Fakultät für Physik, Universität Bielefeld, D-33615 Bielefeld, Germany 
The first and widely discussed probe in this context is the $\mathrm{J} / \psi$. Contrary to naive expectations, Matsui and Satz suggested that with increasing cms energy in a heavy-ion collision a suppression of the $\mathrm{J} / \psi$ production relative to the (DrellYan) continuum occurs due to the dissociation of the charmonium bound state in a dense medium. This is in analogy to the in semiconductor and plasma physics where under high pressure electrons become delocalized and a conduction band emerges, signaling the plasma state [3].

Correlators of quarkonia states are measured at finite temperature on the lattice [4-8]. Using the maximum entropy method [4], spectral functions can be obtained which give not only information about the mass but also about the spectral width of the states. No substantial modification of $\mathrm{J} / \psi$ spectral function is found for temperatures up to $T=1.5 T_{c}$. The $\chi_{c}$ and $\psi^{\prime}$ states may be dissolved at $T_{c}$. This new lattice analysis provides results alternative to those obtained earlier by solving the bound state Schrödinger equation for a (temperaturedependent) screened potential [9]. While the Mott-dissociation of $\chi_{c}$ and $\psi^{\prime}$ states is confirmed, the observation of a nearly unchanged $\mathrm{J} / \psi$ resonance well above the suspected Mott temperature $T_{J / \psi}^{\mathrm{Mott}}=1.1-1.3 T_{c}$ came as a surprise for simple potential models. The existence of above the Mott transition is, however, a well-known feature displayed, e.g., in analyses of exciton lines in semiconductor plasmas [3]. The picture which emerges from these lattice studies seems to be consistent with a modification of the effective interaction at finite temperatures so that excited states of the charmonium spectrum $\left(\chi_{c}\right.$ and $\left.\psi^{\prime}\right)$ can be dissolved at $T_{c}$ whereas the deeply bound ground states $\left(\eta_{c}\right.$ and $\left.\mathrm{J} / \psi\right)$ are observed as rather narrow resonances well above that temperature. In order to interpret these findings a study of the spectral functions for screened Coulomb or Cornelltype potentials should be performed.

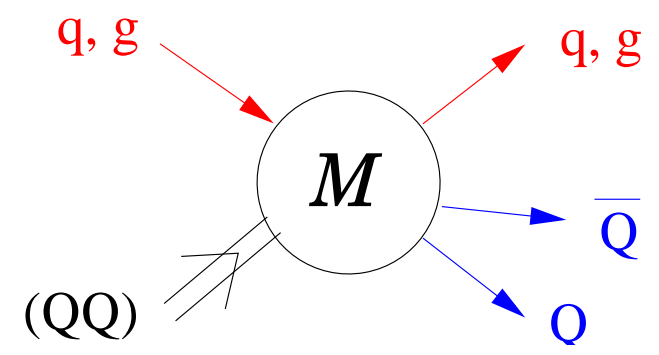

Fig. 1. Transition amplitude for a quarkonium breakup process in a fully developed quark-gluon plasma.

\section{Quantum kinetics for quarkonium in a plasma}

The inverse lifetime of a state with 4-momentum $p$ in a plasma is related to the imaginary part of its selfenergy by $\tau^{-1}(p)=\Gamma(p)=\Sigma^{>}(p)-\Sigma^{<}(p)$, where in 
the Born approximation for the quarkonium breakup by quark and gluon impact holds, see also Fig. 1

$$
\Sigma^{<}(p)=\int_{p^{\prime}} \int_{p_{1}} \ldots \int_{p_{3}}(2 \pi)^{4} \delta_{p+p^{\prime}, p_{1}+p_{2}+p_{3}}|\mathcal{M}|^{2} G^{<}\left(p^{\prime}\right) G^{>}\left(p_{1}\right) G^{>}\left(p_{2}\right) G^{>}\left(p_{3}\right),
$$

where the nonequilibrium Green functions can be expressed via distribution functions $f_{i}(p)$ and spectral functions $A_{i}(p)$ of the particle species $i$ as $G_{i}^{>}(p)=$ $\left[1 \pm f_{i}(p)\right], G_{i}^{<}(p)=f_{i}(p) A_{i}(p)$. This quantum kinetic formulation includes not only the loss but also the reverse process of $Q \bar{Q}$ fusion in a consistent way. It can also be generalized to include strong correlations such as hadronic bound states or resonances in the visinity of the hadronization transition, see Fig. 2. First steps in this direction have been explored [10].

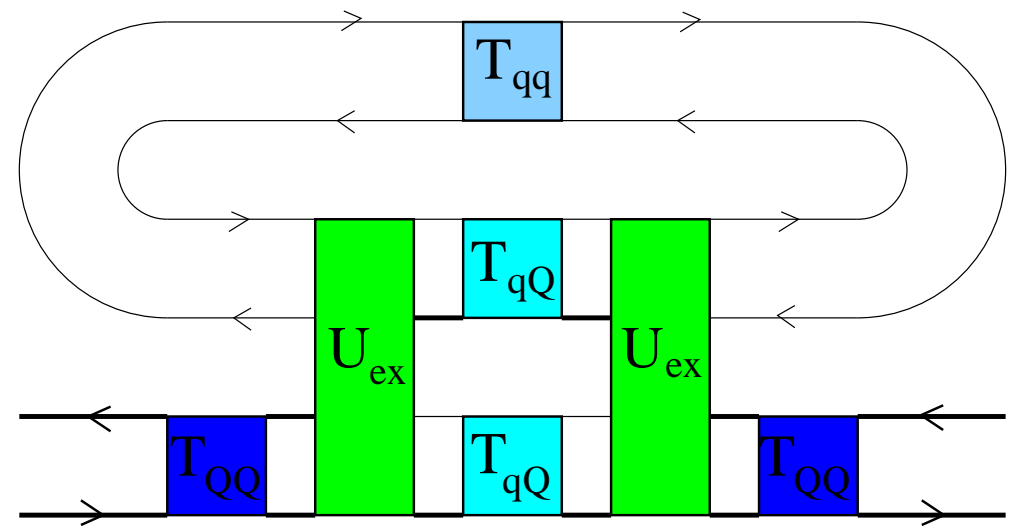

Fig. 2. Transition amplitude for heavy quarkonium dissociation in strongly correlated quark matter. The T-matrices stand for mesonic bound and/or scattering states.

\section{Quarkonium dissociation cross section in a gluon gas}

In this section we give benchmark results for activation of Coulombic bound states by collisions in a medium. The medium will be represented as a gluon gas with thermal distribution function, $n_{g}(\omega)=g_{g}[\exp (\omega(p) / T)-1]^{-1}$, where the degeneracy factor $g_{g}=2\left(N_{c}^{2}-1\right)$.

The quarkonium breakup cross section by gluon impact can be estimated with the Bhanot/Peskin formula [11]

$$
\sigma_{(Q \bar{Q}) g}(\omega)=\frac{2^{11}}{3^{4}} \alpha_{s} \pi a_{0}^{2} \frac{\left(\omega / \epsilon_{0}-a(T)\right)^{3 / 2}}{\left(\omega / \epsilon_{0}\right)^{5}} \Theta\left(\omega-\epsilon_{0} a(T)\right)
$$

with the binding energy $\epsilon_{0}$ of the $1 S$ quarkonium state with a Coulombic rms radius $\sqrt{\left\langle r^{2}\right\rangle_{1 S}}=\sqrt{3} a_{0}=2 \sqrt{3} /\left(\alpha_{s} m_{Q}\right)$, and the heavy-quark mass $m_{Q}$, the 
David Blaschke et al.

\begin{tabular}{|c|cc|cc|}
\hline & \multicolumn{2}{|c|}{ set(i) } & \multicolumn{2}{|c|}{ set(ii) } \\
\hline$Q Q$ system & $\varepsilon_{0}[\mathrm{GeV}]$ & $m_{Q}[\mathrm{GeV}]$ & $\varepsilon_{0}[\mathrm{GeV}]$ & $m_{Q}[\mathrm{GeV}]$ \\
\hline bottomonium & 0.75 & 5.10 & 1.10 & 5.28 \\
charmonium & 0.78 & 1.94 & 0.62 & 1.86 \\
\hline
\end{tabular}

Table 1. Parameters of the heavy quarkonium systems: binding energy $\varepsilon_{0}$ and heavy quark mass $m_{Q}$ from [12]. Left two columns refer to set(i), the right two columns to $\operatorname{set}($ ii).

energy of the impacting gluon is $\omega$. With the parameter $0<a(T)<1$ we can vary the effective dissociation threshold from $\omega_{t h}^{\text {ideal }}=\epsilon_{0} ; a\left(T<T_{c}\right)=1$ to $\omega_{t h}^{M o t t}=0 ; a\left(T>T^{M o t t}\right)=0$. See Fig. 3 for numerical examples.

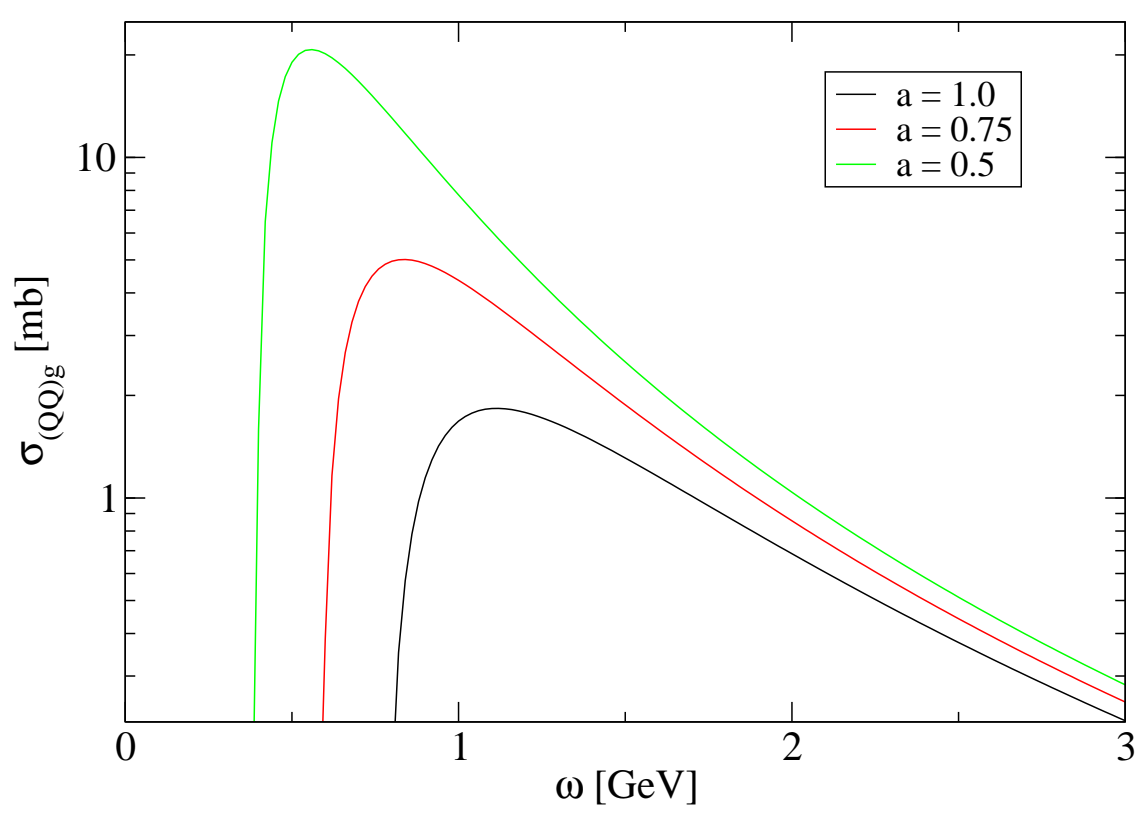

Fig. 3. Energy dependence of the quarkonium breakup cross section by gluon impact for different (constant) threshold depletion factors $a(T)=1,0.75,0.5$. 
The dissociation rate for a heavy quarkonium $1 S$ state at rest in a heat bath of massless gluons $(\omega=p)$ at temparature $T$ is

$$
\frac{1}{\tau_{(Q \bar{Q}) g}(T)}=\Gamma_{(Q \bar{Q}) g}(T)=\left\langle\sigma_{(Q \bar{Q}) g}^{\text {ideal }}(\omega) n_{g}(\omega)\right\rangle_{T}=\frac{1}{2 \pi^{2}} \int_{0}^{\infty} \omega^{2} d \omega \sigma_{(Q \bar{Q}) g}^{\text {ideal }}(\omega) n_{g}(\omega)
$$

and is shown in Fig. 4.

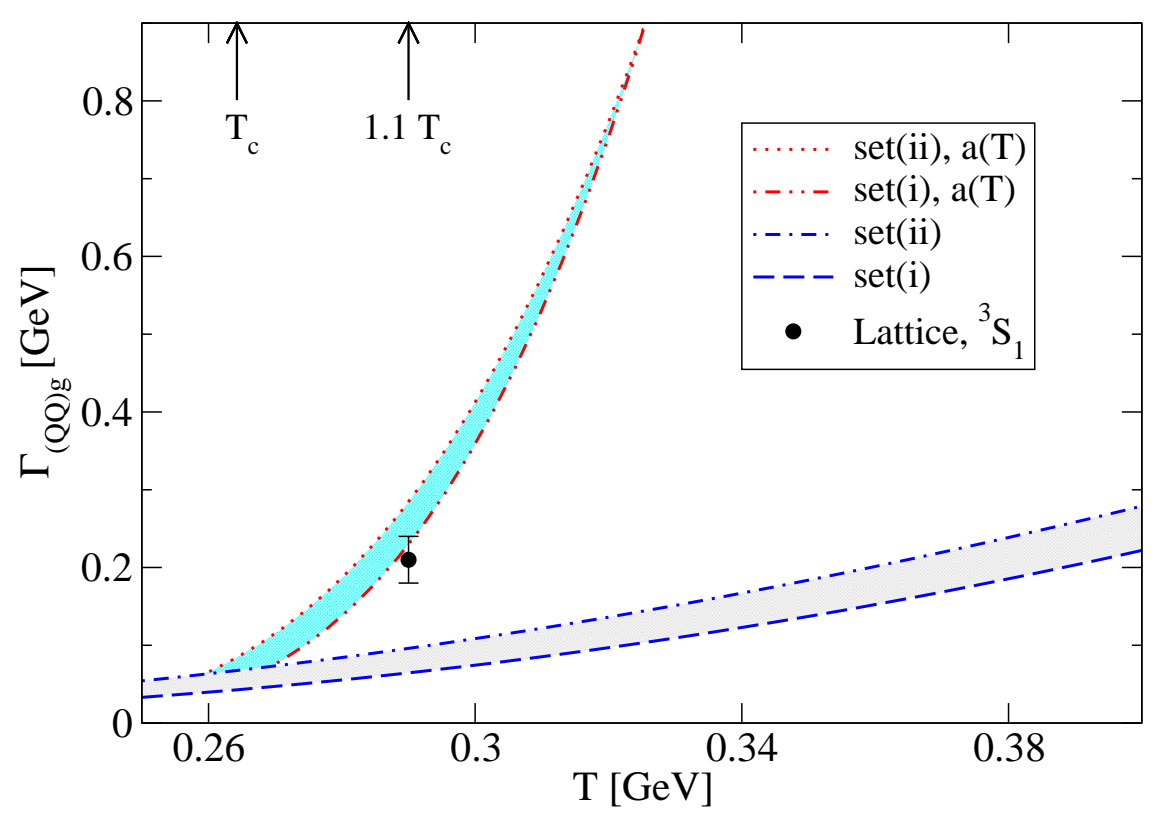

Fig. 4. Rate coefficient $\Gamma_{(Q \bar{Q}) g}=\tau^{-1}$ for heavy quarkonium dissociation by gluon impact as a function of the gluon plasma temperature (no medium, $a(T)=1$ ), parameters see Tab. 1. For comparison, the Breit-Wigner fit of the spectral width for ${ }^{1} S_{0}$ charmonium from lattice simulations is shown. In-medium effects are estimated by a lowering of the breakup threshold, see text.

It is interesting to note that there is a discrepancy between the Lattice calculation of the charmonium decay with in a gluonic medium and the decay width of charmonium by gluon impact due to the gluonic E1 transition. It is conceivable that the Lattice simulation overestimates the thermal width of the charmonium states, but we would rather like to suggest that due to a lowering of the ionisation threshold in the gluonic medium the breakup rate will be enhanced. For an analytical estimate we adopt a temperature dependence of the strong coupling 
constant by using the gluon momentum scale $[13]\left\langle p^{2}\right\rangle=(3.2 T)^{2}$ in the 1-loop $\beta$ function such that

$$
\frac{\varepsilon_{0} a(T)}{m_{Q}}=\left(\frac{3 \pi}{22 \ln (3.2 T / \Lambda)}\right)^{2} .
$$

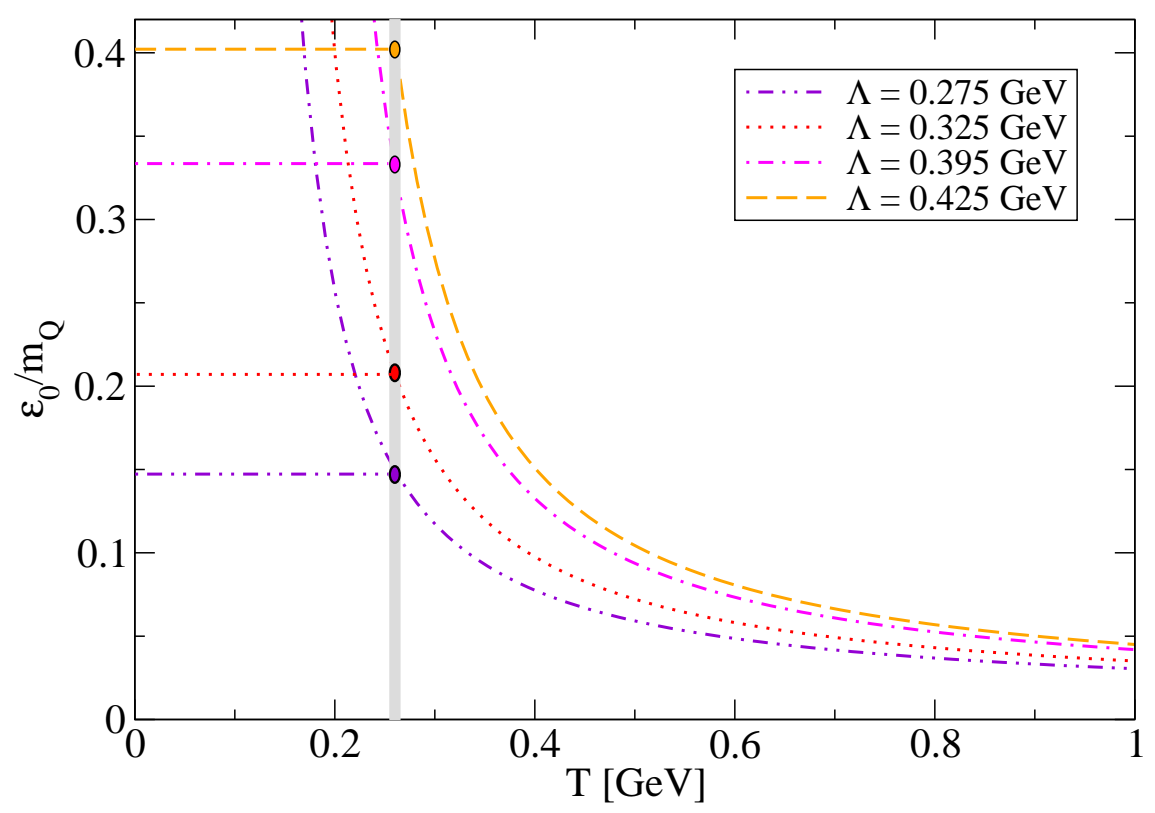

Fig. 5. Temperature dependence of the ionisation threshold due to the 1-loop running of the strong coupling constant (3).

\section{Quarkonia abundances and observable signatures}

In order to study observable signatures we will adopt here the Bjorken scenario [14] for the plasma evolution, i.e. longitudinal expansion with conserved entropy: $T^{3} t=T_{0}^{3} t_{0}=$ const. Parameters for the initial state are given in Tab. 2, where RHIC and LHC values are taken from [15], the SPS values are "canonical". As the quantity for comparison with experimental data of quarkonium production we consider the survival probability. We neglect here the hadronic comover and the nucleonic contributions, and also the effects due to the hadronisation phase transition

$$
S\left(t_{f}\right)=\exp \left(-\int_{t_{0}}^{t_{f}} d t \tau^{-1}(T)\right)
$$




\begin{tabular}{|c|c|c|c|}
\hline & LHC (3 & $\mathrm{HIC}$ & SPS \\
\hline$T_{0}[\mathrm{GeV}]$ & 0.72 & 0.4 & 0.25 \\
\hline$\tau_{0}[\mathrm{fm} / \mathrm{c}]$ & 0.5 & 0.7 & 1.0 \\
\hline
\end{tabular}

Table 2. Heavy-ion collision parameters from [15].

At the freeze-out time $t_{f}$ the collisions stop to change the number of $\mathrm{J} / \psi(\Upsilon)$. Using the Bjorken scaling it can be related into a freeze-out temperature. In Fig. 6 we give the survival probability for $\mathrm{J} / \psi$ (left panel) and for $\Upsilon$ (right panel) for the parameters of the Tables 1 and 2 due to gluon impact. For the LHC conditions, the simple estimates presented here don't give robust predictions for $\mathrm{J} / \psi$. The $\Upsilon$, however, shall be a good probe for the lifetime of the plasma state as well as for its temperature. More details can be found in Ref. [17].

\section{Quarkonia dissociation by quark impact, $T>T_{c}$}

The quarkonium breakup cross section by quark impact is estimated using the Bethe formula [16] for impact ionization of the $1 S$ bound state of the Coulomb potential (the quark now plays the role of the impacting electron)

$$
\sigma_{(Q \bar{Q}) q}(\omega)=2.5 \pi a_{0}^{2} \frac{\varepsilon_{0}}{\omega} \ln \left(\frac{\omega+\Delta(T)}{\varepsilon_{0}}\right) \Theta\left(\frac{\omega+\Delta(T)}{\varepsilon_{0}}-1\right) .
$$

Here we employ a generalization of Bethe's formula which takes into account the shift of the continuum edge of scattering states by $\Delta(T)$, which results in a temperature-dependent lowering of the ionisation threshold [23], $\varepsilon(T)=$ $\varepsilon_{0}-\Delta(T)$. This is named the Bethe-Born model (BBM). For quark matter systems, the energy shift $\Delta(T)$ has been obtained within the string-flip model (SFM), see $[18,19]$. Within this model also an effective cross section for heavy quarkonium breakup has been estimated $[20,21]$

$$
\sigma(T)=\pi r_{Q \bar{Q}}^{2}(T) \exp (-\Delta(T) / T)
$$

It is remarkable that the enhancement of the breakup cross section due to the lowering of the threshold in the BBM is comparable in magnitude to that of the SFM approach, see Fig. 7 .

\section{Conclusions}

In the QGP (and in the mixed phase), due to the presence of quasifree quarks and gluons, new channels for charmonium formation and dissociation exist which could drive chemical equilibration during the existence of the fireball formed in the heavy-ion collision. In this contribution we have given some benchmark estimates which have to be further elaborated. The role of partonic in-medium effects in the heavy quarkonium kinetics in a QGP, which has previously been 

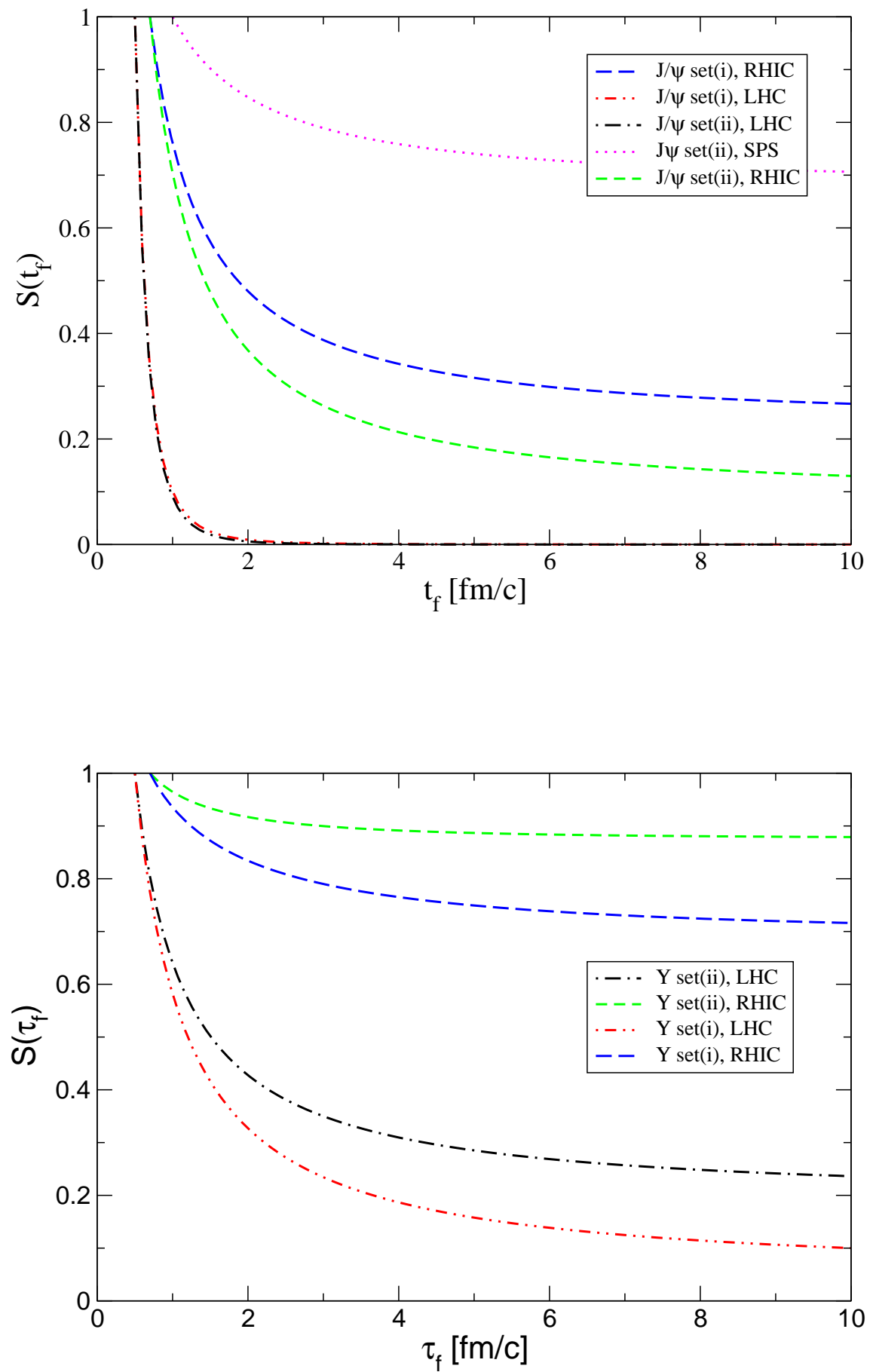

Fig. 6. Survival probability for heavy quarkonia states in a longitudinally expanding gluon plasma as a function of the plasma lifetime; ${ }^{1} S_{0}$ charmonium $(\mathrm{J} / \psi$, upper panel) and ${ }^{1} S_{0}$ bottomonium ( $\Upsilon$, lower panel), parameters see Tabs. 1, 2. 

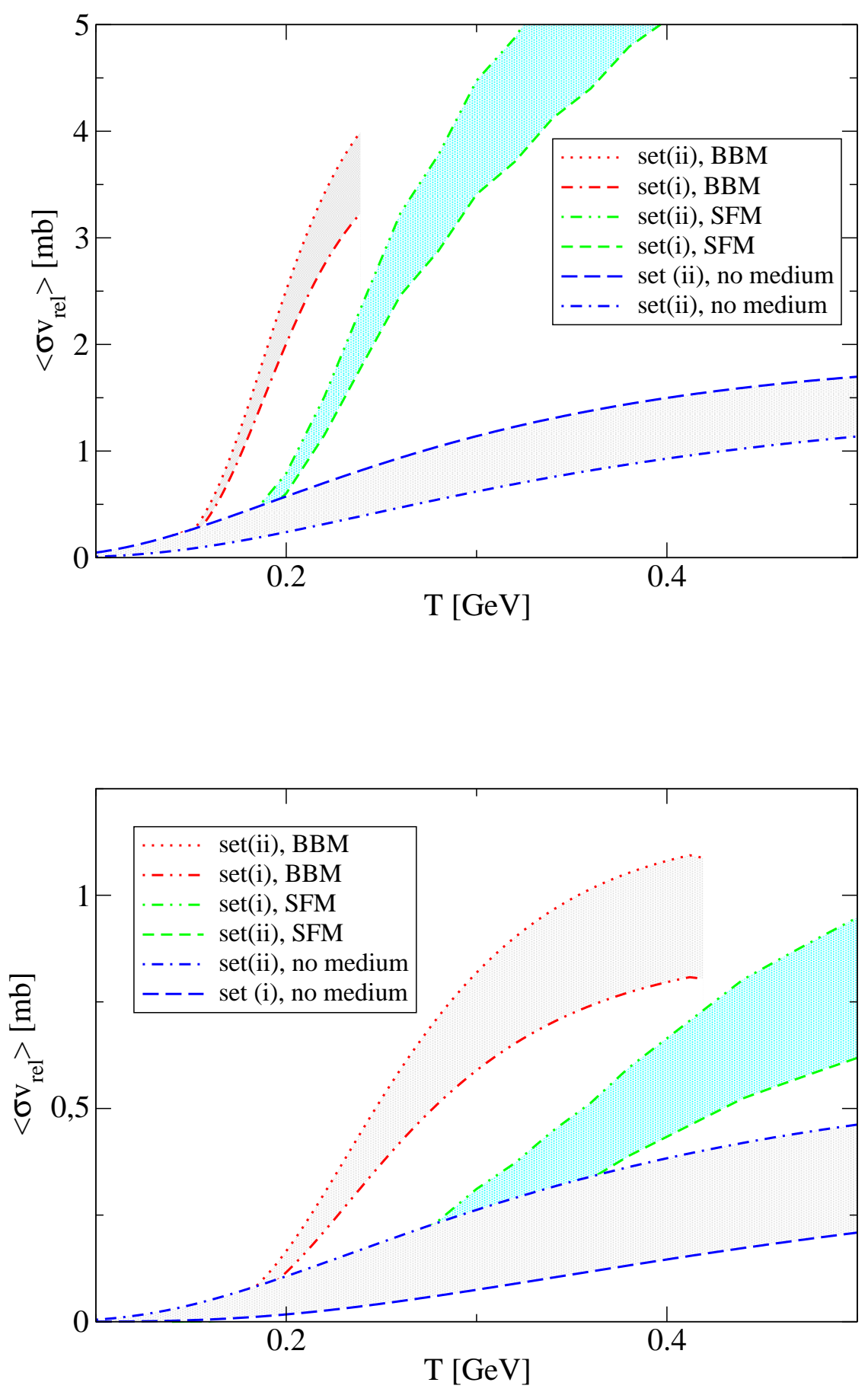

Fig. 7. Thermally averaged cross section for heavy quarkonium dissociation by quark impact as a function of the temperature: $\mathrm{J} / \psi$ (upper panel) and $\Upsilon$ (lower panel), parameters see Tab. 1. BBM and SFM approach give a similar cross section enhancement due to the lowering of the breakup threshold. 
discussed in the string-flip model of quark matter in the form of a modified mass action law [20] and dissociation rate [21], should be reconsidered by also including gluonic degrees of freedom which shall become dominant at RHIc and LHC conditions. We suggest that rate coefficients for the ionization and recombination of charm mesons could be described using an approach similar to methods used previously to study Coulomb plasmas $[3,22,23]$. It is suggested that the measurement of the $\Upsilon$ suppression at LHC can provide rather robust informations about the initial temperature (or lifetime) of the quark-gluon plasma.

\section{Acknowledgement}

V.Yu. acknowledges support by DFG under grant No. 436 RUS 17/37/03 and the Russian Fund for Fundamental Research under grant No. 02-02-16194 as well as by the Heisenberg-Landau program. D.B. is grateful to many colleagues for numerous enlightening discussions, in particular to G. Burau, J. Hüfner, P. Petreczky, A. Polleri, H. Satz and R. Vogt.

\section{References}

1. T. Matsui and H. Satz, Phys. Lett. B 178 (1986) 416.

2. K. Kajantie, Quark Matter '84. Proceedings, 4th International Conference On Ultrarelativistic Nucleus-Nucleus Collisions, Helsinki, Finland, June 17-21, 1984,

3. R. Redmer, Phys. Rep. 282 (1997) 35

4. Y. Nakahara, M. Asakawa and T. Hatsuda, Phys. Rev. D60 (1999) 091503

5. M. Asakawa, T. Hatsuda and Y. Nakahara, Prog. Part. Nucl. Phys. 46 (2001) 459

6. T. Umeda, R. Katayama, O. Miyamura and H. Matsufuru, Int. J. Mod. Phys. A 16 (2001) 2215

7. T. Umeda, K. Nomura and H. Matsufuru, hep-lat/0211003

8. S. Datta, F. Karsch, P. Petreczky and I. Wetzorke, hep-lat/0208012 (to be published in the proceedings of Lattice 2002)

9. F. Karsch, M.T. Mehr and H. Satz, Z. Phys. C (1988) 617

10. G. R. G. Burau, D. B. Blaschke and Y. L. Kalinovsky, Phys. Lett. B 506 (2001) 297 [arXiv:nucl-th/0012030].

11. G. Bhanot and M.E. Peskin, Nucl. Phys. B 156 (1979) 391

12. F. Arleo, P.-B. Gossiaux, T. Gousset, J. Aichelin, Phys. Rev. D 65 (2001) 014005

13. J.I. Kapusta, Finite-temperature field theory, Cambridge University Press, Cambridge 1989

14. J. D. Bjorken, Phys. Rev. D 27 (1983) 140

15. X.-M. Xu, D. Kharzeev, H. Satz, X.-N. Wang, Phys. Rev. C 53 (1996) 3051

16. H. Bethe, Ann. Phys. (Leipzig) 5 (1930) 325

17. M. Bedjidian et al., arXiv:hep-ph/0311048.

18. D. Blaschke, F. Reinholz, G. Ropke and D. Kremp, Phys. Lett. B 151 (1985) 439.

19. G. Ropke, D. Blaschke and H. Schulz, Phys. Rev. D 34 (1986) 3499.

20. G. Röpke, D. Blaschke, and H. Schulz, Phys. Lett. B 202 (1988) 479.

21. G. Röpke, D. Blaschke and H. Schulz, Phys. Rev. D 38 (1988) 3589

22. Th. Bornath and M. Schlanges Physica A 196 (1993) 427.

23. M. Schlanges, Th. Bornath, D. Kremp, Phys. Rev. A 38 (1988) 2174. 